\title{
Photoionization of helium atoms irradiated with intense vacuum ultraviolet free-electron laser light. Part I. Experimental study of multiphoton and single-photon processes
}

\author{
T. Laarmann, ${ }^{1, *}$ A. R. B. de Castro, ${ }^{2}$ P. Gürtler, ${ }^{1}$ W. Laasch, ${ }^{1}$ J. Schulz, ${ }^{1, \dagger}$ H. Wabnitz, ${ }^{1, \$}$ and T. Möller ${ }^{1, \S}$ \\ ${ }^{1}$ Hamburger Synchrotronstrahlungslabor HASYLAB at Deutsches Elektronen Synchrotron DESY, \\ Notkestr. 85, 22607 Hamburg, Germany \\ ${ }^{2}$ Laboratorio Nacional de Luz. Sincrotron LNLS 13084-971 Campinas SP, Brazil and Instituto de Fisica Gleb Wataghin, \\ Universidade Estadual de Campinas IFGW-UNICAMP 13083-970 Campinas SP, Brazil
}

(Received 30 August 2004; published 15 August 2005)

\begin{abstract}
The interaction of $\mathrm{He}$ atoms with intense vacuum-ultraviolet light of a free-electron laser is investigated using time-of-flight mass spectroscopy and photoelectron spectroscopy. The atoms were irradiated with $100 \mathrm{fs}$ pulses at $95 \mathrm{~nm}$ wavelength, which corresponds to $\sim 13 \mathrm{eV}$ photon energy. The ionization of $\mathrm{He}$ atoms is observed at a peak intensity of $10^{10}-10^{13} \mathrm{~W} / \mathrm{cm}^{2}$, which is due both to nonlinear multiphoton ionization with the fundamental wavelength and single-photon ionization with third harmonic radiation of the free-electron laser. The observation of two sharp photoelectron peaks in the kinetic energy spectra, that are separated by the photon energy, is in agreement with the numerical solution of the time-dependent Schrödinger equation. The calculation was done using the fully quantized field and a limited but representative set of basis states. The ionization rate dependence on the laser peak intensity indicates that: (a) The low-energy peak in the photoelectron spectra is mainly due to two-photon absorption of the fundamental, but (b) the high-energy peak at $15.4 \mathrm{eV}$ is probably due to third harmonic FEL radiation. The theoretically predicted contribution from threephoton absorption of the fundamental is of about the same order of magnitude and could not be separated from the third harmonic background signal. Particularly, the photoelectron spectra and $\mathrm{He}^{+}$time-of-flight data give evidence that the intensity of third harmonic light is high enough to perform single-shot spectroscopy on gas phase samples.
\end{abstract}

DOI: $10.1103 /$ PhysRevA.72.023409

PACS number(s): 42.50.Hz, 33.60.Cv, 32.80.Rm

\section{INTRODUCTION}

With the discovery of above-threshold ionization (ATI) in 1979 by Agostini et al. [1], tremendous effort both experimentally and theoretically has begun in order to investigate highly nonlinear phenomena in rare gas atoms irradiated with intense $\left(I \geqslant 10^{14} \mathrm{~W} / \mathrm{cm}^{2}\right)$ fs-laser pulses at optical frequencies [2]. In general, ATI describes a process in which the atom, respectively, the electron, absorbs more laser photons than necessary to overcome the ionization potential. This results in a kinetic energy distribution of photoelectrons, which exhibit a characteristic series of equally spaced lines separated by the photon energy. These phenomena gave origin to a semiclassical model of electron motion in an oscillating electric field, which, by now, is experimentally well established for laser fields in the infrared (IR) and visible spectral ranges. In this picture an important parameter is the ponderomotive energy (the average kinetic energy the electron can gain from the oscillating field). At optical laser frequencies and intensities of the order of $10^{14} \mathrm{~W} / \mathrm{cm}^{2}$, the ponderomo-

\footnotetext{
*Present address: Max-Born-Institute, Max-Born Str. 2a, D-12489

Berlin, Germany. Electronic address: tim.laarmann@desy.de

${ }^{\dagger}$ Present address: MAX-lab, Box 118, 22100 Lund, Sweden.

†Present address: CEA-SPAM, Bât. 522, Centre d'Etudes de Saclay, 91191 Gif-sur-Yvette, France.

${ }^{\S}$ Present address: Technische Universität Berlin, Hardenbergstr. 36, D-10623 Berlin, Germany.
}

tive force is close to $10 \mathrm{eV}$ and it sets the energy scale for the maximum energy of the released electrons [3-6]. The distribution of photoelectrons with kinetic energy much higher than the ponderomotive energy is a subject of current interest $[3,6-8]$.

In this context, it is interesting to investigate the atomic response to intense short-wavelength radiation in the VUV spectral range, where concepts like field-ionization become less relevant because the ponderomotive energy is very small. More specifically, it is interesting to know if details of the atomic structure, such as electronic shells, orbitals, or excited intermediate states affect ionization in the limit, where the radiation frequency is of the same order of magnitude, or larger, than the characteristic frequency of electron orbital motion. The pioneering work on rare gas atoms in the direction of short wavelength was done by Rhodes and coworkers almost 20 years ago using $\mathrm{ArF}^{*}$ and $\mathrm{KrF}^{*}$-lasers operating at 193 and $248 \mathrm{~nm}$ wavelength, respectively. At a laser intensity up to $10^{15} \mathrm{~W} / \mathrm{cm}^{2}$ and a pulse duration of $\sim 0.5-5$ ps various aspects of nonlinear effects were investigated $[11,12]$.

The output from the free-electron laser (FEL) working after the Self-Amplified-Spontaneous-Emission (SASE) principle of the TESLA Test Facility (TTF) at DESY has recently opened the door to a new regime of strong field matter interaction at wavelength below $100 \mathrm{~nm}$ in the vacuum-ultraviolet (VUV) spectral range [13]. First experiments were performed on xenon and argon clusters, which have already thrown up some surprises, namely (i) very ef- 


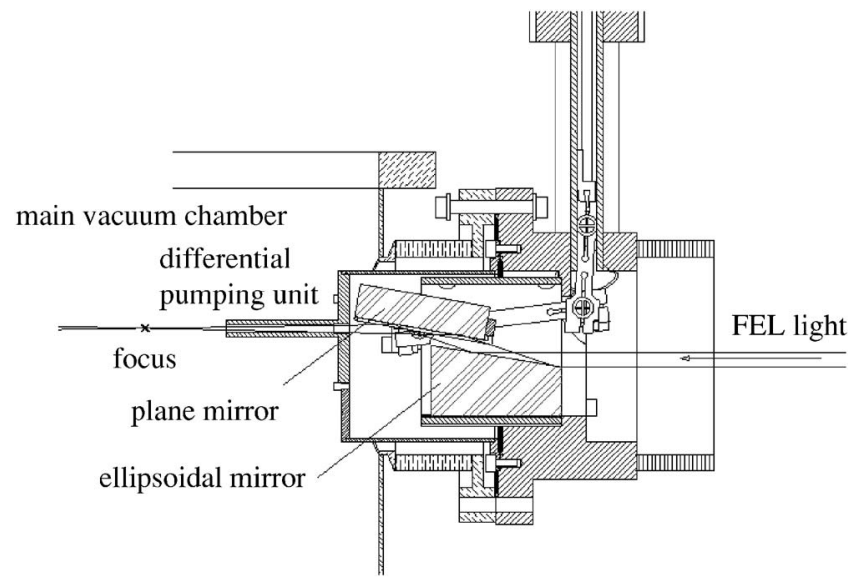

FIG. 1. Longitudinal section of the mirror chamber. A small tube (diameter $2 \mathrm{~mm}$ ) separates the mirror chamber from the main chamber. The adjustment of both mirrors was remote controlled.

ficient energy absorption and (ii) ionization by thermally activated electron emission, which is different from the dominant process at optical frequencies [14-17].

In this contribution, we present time-of-flight mass spectra and photoelectron spectra of $\mathrm{He}$ atoms irradiated with Gigawatt VUV-FEL light pulses of $\sim 100$ fs length at a repetition rate of $1 \mathrm{~Hz}$ [13]. The paper is organized in the following way. In Sec. II, we describe the experimental setup for investigation of multiphoton ionization of He atoms using time-of-flight techniques. In Sec. III, the experimental results are presented. In this chapter, we first discuss mass spectra and analyze the $\mathrm{He}^{+}$-ionization rate as a function of the peak intensity (Sec. III A). The second part concentrates on the photoemission studies (Sec. III B).

\section{EXPERIMENT}

The VUV-FEL radiation $\left(95 \mathrm{~nm}, h \nu_{\mathrm{FEL}} \sim 13 \mathrm{eV}\right)$ with typical pulse energies between 0.3 and $5 \mu \mathrm{J}$ was focused on a beam of He atoms using an assembly of an elliptical and a plane mirror (Au-coated) at grazing incidence, with a focal distance of $200 \mathrm{~mm}$, as shown in Fig. 1. At the undulator exit the radiation spot size was $250 \mu \mathrm{m}$ [full width at half maximum (FWHM)] with an angular divergence of $260 \mu \mathrm{rad}$ [18]. After $13 \mathrm{~m}$ (source distance) the radiation spot was enlarged to $\sim 3.4 \mathrm{~mm}$ (FWHM). By measuring the focal spot on a $\mathrm{ZnSCu}$-coated fluorescence screen with a microscope and a CCD-camera, a diameter of $\sim 20 \mu \mathrm{m}$, was derived. The calculated depth of focus is $2 \mathrm{~mm}$. Given the $20 \mathrm{~mm}$ focal diameter, a peak intensity up to $\sim 10^{13} \mathrm{~W} / \mathrm{cm}^{2}$ was estimated. In a SASE-FEL, the radiation energy strongly fluctuates from pulse to pulse. The fluctuations are inherent to the SASE process and result from start of the amplification process from shot noise. For online monitoring of the pulse energy a microchannel plate (MCP) based detector was used. The setup consisted of a thin gold wire mesh that scattered a tiny fraction of FEL radiation onto the MCP. Despite its characteristic nonlinear gain as a function of supply voltage, careful calibration of the MCP allowed for absolute measurements of the radiation energy in the laser pulse on a shot-to-shot basis [19].

We would like to note, that an intrinsic property of FEL radiation is the occurrence of higher-harmonics of the fundamental laser frequency. If a high-quality electron beam passes a planar undulator with a strong magnetic field, spontaneous emission at the fundamental resonant frequency and its higher harmonics induce a modulation of the electron charge distribution at their respective wavelength scales. This so-called "micro-bunching" leads to amplified emission. Here, the linear amplification of the higher harmonics is always much smaller than the fundamental and moreover, only odd harmonics are emitted on-axis [20]. The intensity of higher harmonic components is approximately proportional to the intensity at the fundamental laser frequency [21].

The pulsed beam of He atoms was prepared in a gas expansion at a stagnation pressure of 5 bar through a conical nozzle (diameter $100 \mu \mathrm{m}$, half opening cone angle $15^{\circ}$ ), which was mounted on a solenoid valve. The opening time of the nozzle was $2 \mathrm{~ms}$ at a frequency of $1 \mathrm{~Hz}$ synchronized with the repetition rate of the VUV-FEL. In order to prepare a well-collimated beam for time-of-flight experiments, a skimmer $\left(1 \mathrm{~mm}, 25^{\circ}\right)$ was installed in a distance of $75 \mathrm{~mm}$ from the laser focus. The electrons and ions produced during the laser atom interaction were detected in the direction of the laser polarization with the MCP of a bi-polar time-offlight (TOF) spectrometer. The name of this detector originates from its ability to detect either positive ions or negative electrons. Thereby, the output polarity is maintained at ground potential by electro-optically isolating the signal. This is realized by converting the electron avalanche from the MCP into photons in a scintillator and then reconverting them into electrons in a photomultiplier tube, whose signal output is kept at ground potential. Mass spectra of He ions resulting from an atomic beam and photoelectron spectra recorded with the bi-polar TOF are discussed in Secs. III A and III B, respectively. For the benefit of photoelectron flighttime/kinetic energy resolution, a $150 \mathrm{~mm}$ long field-free drift tube is provided. The grounded tube is made of $\mu$-metal in order to reduce the influence of magnetic fields on the flight path of the electrons. To avoid surface charging, the tube and the accelerating plate are coated with gold. The acceptance angle of the detector was limited to $6^{\circ}$ by a $2 \mathrm{~mm}$ entrance aperture in front of the drift tube. In order to increase the collection efficiency for photoelectrons, an extraction voltage of $-10 \mathrm{~V}$ was applied between the grounded aperture and an acceleration plate mounted in a distance of $12 \mathrm{~mm}$ with the laser focus in between. The photomultiplier was coupled to a fast sampling oscilloscope reading out the analog signal. The measured electron distribution curve $d I / d t$ was converted into kinetic energy spectra $d I / d E$, recalling that $d I / d E$ $\sim t^{3} d I / d t$, and corrected with respect to the calculated transmission curve of the spectrometer. Due to the extraction voltage between the acceleration plate and the aperture, the transmission of the spectrometer increases by approximately three orders of magnitude for electrons with a kinetic energy below $\sim 2.5 \mathrm{eV}$ as shown in Fig. 2. The settings of the bipolar TOF for ion and electron detection are given in Table I. Investigation of multiphoton ionization as a function of the VUV-FEL peak intensity was realized by either selecting 


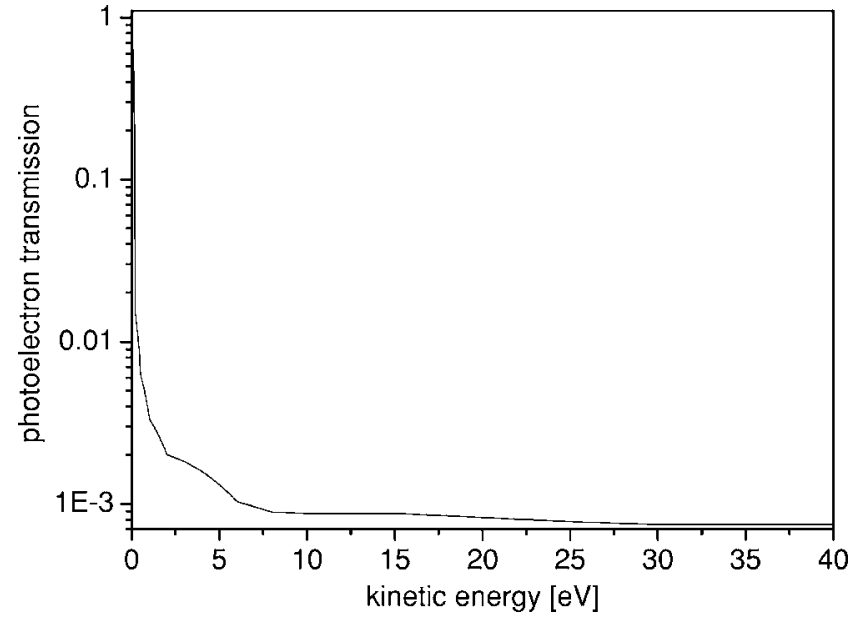

FIG. 2. Transmission curve of photoelectrons as a function of their kinetic energy.

photoelectron spectra or mass spectra of He atoms irradiated with laser pulses within a specific energy bin or by shifting the focus of the VUV light beam to a position $d$ outside the volume $V_{\text {detect }}$ seen by the TOF. This results in a decrease of the peak intensity by a geometric factor $k(d)$ which is given in Table II for different displacements of the focus. It should be noticed at this point that even for the largest value of $d$ used, all photons $N_{p h}$ in the FEL pulse still cross $V_{\text {detect }}$. In addition, since the dimension of the region seen by the TOF is comparable to the depth of focus, the shape of the interaction region is approximately cylindrical with radius $r$ for all choices of the displacement $d$ and assuming a Gaussian beam profile the light intensity in this cylinder is given by:

$$
I(d, r)=I_{\text {center }}(d) \exp \left(\frac{-r^{2}}{2 \sigma(d)^{2}}\right)
$$

\section{RESULTS AND DISCUSSION}

\section{A. Mass spectra of helium atoms}

Figure 3 shows a characteristic TOF mass spectrum of a beam of He atoms, which was obtained by averaging over several dozens of laser shots within a $\pm 6 \%$ laser energy bin. The spectrum, recorded at a peak intensity of $\sim 8 \cdot 10^{12} \mathrm{~W} / \mathrm{cm}^{2}$ and $13 \mathrm{eV}$ photon energy, shows that the $\mathrm{He}$ atoms are efficiently ionized by intense VUV-FEL radia-

TABLE I. Settings of the bi-polar time-of-flight detector for $\mathrm{He}$ ion and electron detection.

\begin{tabular}{ccc}
\hline \hline Applied voltages & Ion detection & Electron detection \\
\hline acceleration plate $[\mathrm{V}]$ & +1000 & -10 \\
drift tube & grounded & grounded \\
MCP input $[\mathrm{kV}]$ & -2.6 & grounded \\
MCP output $[\mathrm{kV}]$ & -1.8 & +1.0 \\
gain control $[\mathrm{kV}]$ & +0.2 & +3.0 \\
photomultiplier $[\mathrm{kV}]$ & -0.6 & -0.6 \\
\hline \hline
\end{tabular}

TABLE II. Reduction of the laser peak intensity by a geometric factor $k$ for the same pulse energy but different distances $d[\mathrm{~mm}]$ of the recipient outside the focus.

\begin{tabular}{cc}
\hline \hline$d[\mathrm{~mm}]$ & $k$ \\
\hline 3 & 0.044 \\
8 & 0.0083 \\
15 & 0.0026 \\
\hline
\end{tabular}

tion. However, a peak related to double-ionization of helium, an issue which has been of great interest over the last few years (see Ref. [22] and references therein), is not observed in the spectrum. $\mathrm{H}_{2} \mathrm{O}^{+}$ions in the mass spectra result from single-photon ionization of residual gas molecules, since the photon energy is well-above the ionization threshold of water.

At optical laser frequencies, ionization occurs through either multiphoton or field ionization (above-barrier or tunnelling). In the perturbative regime, the dominant process can be estimated from the so-called Keldysh parameter $\gamma$, that describes the ratio between tunnelling time and laser period [23]. The Keldysh parameter can be expressed by the electron binding energy $E_{b i}$ and the ponderomotive potential $U_{p}$. As a result, one obtains $\gamma=\left(E_{b i} / U_{p}\right)^{1 / 2}$. If $\gamma$ is smaller than 1 field ionization dominates, whereas for a large Keldysh parameter multiphoton ionization (MPI) is the dominant process.

In case of VUV-laser interaction with He atoms discussed in this article, the experiments are performed in the multiphoton regime ( $\gamma \sim 43$ for FEL-parameters of Fig. 3$)$ and not quite in the perturbative regime. We would like to note that the ionization potential of He atoms is $24.59 \mathrm{eV}$. Thus, there are two possible ionization processes, namely (i) multiphoton ionization of neutral $\mathrm{He}$ atoms with the first harmonic of the FEL ( $\sim 13 \mathrm{eV}$ photons), or (ii) single-photon ionization with the third harmonic ( $\sim 39 \mathrm{eV}$ photons). The contribution of third harmonic radiation within the laser pulse is theoretically estimated to be less than $0.1 \%$ of the fundamental

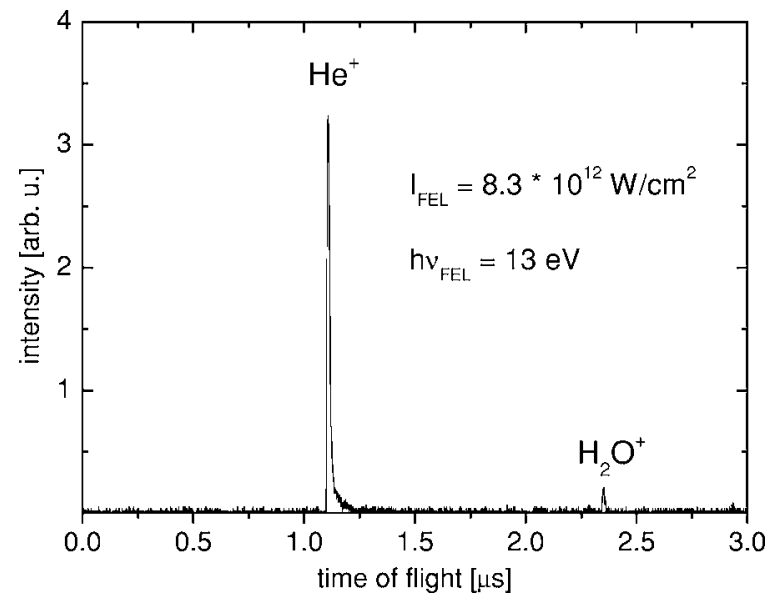

FIG. 3. Ionic products after irradiation of an atomic beam of helium. The FEL parameters are given in the figure. $\mathrm{H}_{2} \mathrm{O}^{+}$ions result from single-photon ionization of residual gas molecules. 
power level [21], but has not yet been measured directly.

To shed light on the basic mechanism of He ionization observed in Fig. 3, we recall that MPI is the multiphoton transition from a bound state to a free (continuum) state [24]. In lowest-order perturbation theory the $n$-photon ionization rate $\Gamma_{n}$ is given by

$$
\Gamma_{n}=\xi_{n} \cdot I^{n}
$$

where $n$ is the minimum number of photons needed for ionization, $\xi_{n}$ is the generalized cross section and $I$ is the incident laser intensity. It should be noticed that this formula is a particular case of the expression

$$
P(I)=\sum_{j} \xi_{j} \cdot I^{j}
$$

which is just a general power series expansion of the transition probability $P(I)$ for arbitrary situations in either a perturbative or nonperturbative, highly nonlinear regime. According to Eq. (3), if one single term in the sum dominates, Eq. (2) applies and the ionization rate of $\mathrm{He}^{+}$should follow a straight line in a double logarithmic diagram $(\log \Gamma-\log I)$ with the slope $n$. This allows identification of the dominant underlying ionization mechanism. More specifically, we can analyze if the detected ions are mainly produced by multiphoton or single-photon ionization in the intensity regime under investigation, or in other words with first or third harmonic VUV-FEL radiation. Therefore, TOF mass spectra of $\mathrm{He}$ atoms similar to that presented in Fig. 3 were recorded at reduced FEL pulse energies and consequently reduced peak intensities in the focal plane. In a second set of measurements the peak intensity was additionally lowered by moving the chamber (i.e., the interaction volume imaged onto the TOF) $8 \mathrm{~mm}$ out of the focus, which results in a decrease by a factor of $k=0.0083$ (see Table II). We would like to note, that the average He target density in the interaction volume, as well as the photon energy $(\sim 13 \mathrm{eV})$ and the pulse duration $(\sim 100 \mathrm{fs})$ were kept constant during the experiments. By normalization of the measured $\mathrm{He}$ ion yield with respect to the size of the interaction volume, we obtained the $n$-photon ionization rate $\Gamma_{n}$ of $\mathrm{He}^{+}$ions as a function of the peak intensity $I$. The experimental data is plotted in Fig. 4 in a $\log \Gamma-\log I$ diagram. The slope of the linear fit is $n \sim 1$, which means the detected ions result mainly from the ionization of $\mathrm{He}$ atoms through single-photon processes by the third harmonic of the VUV-FEL. In order to identify a signature of multiphoton processes to the $\mathrm{He}^{+}$ion yield, we performed additionally a photoemission study on helium atoms. These results will be discussed in the following subsection.

\section{B. Photoelectron spectra of helium atoms}

Photoemission gives a complementary and more detailed view of nonlinear processes of atoms in intense laser fields compared to mass spectroscopic studies. For example, the observation of sharp lines in a photoelectron spectrum clearly reflects multiphoton ionization (MPI), whereas fieldionization results in an exponentially decreasing electron distribution curve starting at $0 \mathrm{eV}$ kinetic energy. Secondly, the

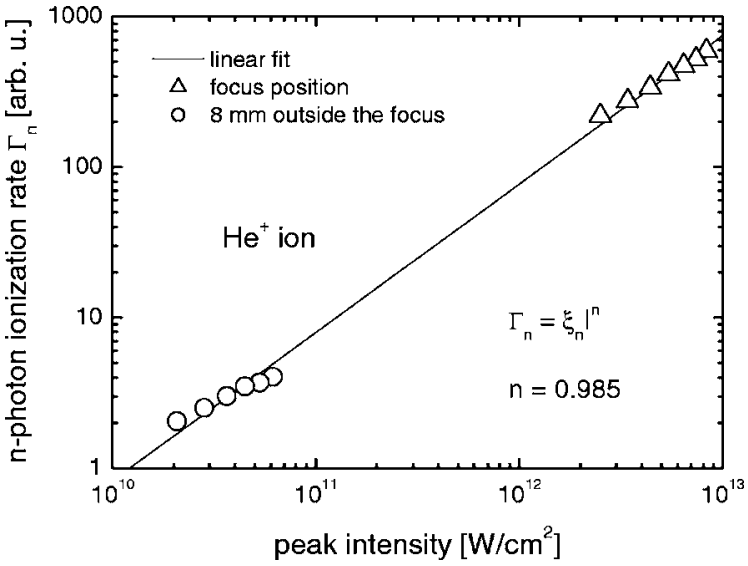

FIG. 4. $n$-photon ionization rate $\Gamma_{n}$ of $\mathrm{He}^{+}$ions as a function of the FEL peak intensity $I$ in a double-logarithmic scale. The slope of the linear fit is approximately $n=1$. The generalized cross section of the ionization process is given by $\xi_{n}$. Data points obtained in the focus (triangles) and at reduced peak intensities $8 \mathrm{~mm}$ outside the focus (circles) are indicated.

kinetic energy distribution curve of photoelectrons in the MPI process is a fingerprint of the number of photons absorbed during the electronic transition. This is simply because the peaks appear at different energy positions separated by the photon energy.

A photoelectron spectrum of $\mathrm{He}$ atoms recorded at a peak intensity of $5.4 \cdot 10^{12} \mathrm{~W} / \mathrm{cm}^{2}$ is shown in Fig. 5(a). The spectrum was obtained by averaging over several dozens of laser shots within a $\pm 27 \%$ laser energy bin, both with and without gas flow in order to subtract the background signal. The resulting spectrum is very noisy, especially at low kinetic energies of the electrons (long flight-times $t$ ). This is due to uncertainties in the background subtraction of spectra with low count rate $d I / d t$, which are then converted into the energy scale. In this context, one has to keep in mind that the transmission of the spectrometer (Fig. 2) increases by approximately three orders of magnitude below a few eV electron energy, which results in efficient collection of lowenergy "stray" electrons originating from apertures, etc. In spite of these experimental difficulties, there are three peaks clearly visible in the photoelectron spectrum of He labeled with A $(0.9 \mathrm{eV}), \mathrm{B}(1.7 \mathrm{eV})$, and C (15.4). The energy resolution of the spectrometer is given by the line widths. Taking into account the ionization potential of He atoms $(24.59 \mathrm{eV})$, the photon energy of the fundamental radiation $(13 \mathrm{eV})$ and that of the third harmonic $(39 \mathrm{eV})$, the peaks A and/or B can be related to two-photon ionization and peak $\mathrm{C}$ to threephoton absorption of the fundamental and single-photon ionization of He atoms with the third harmonic of the VUV-FEL as indicated by vertical lines.

We start with the discussion of peak A. The dependence of peak A on the incident intensity $I$, after taking into due account the varying interaction volume, is shown in Fig. 6(a). The theoretical prediction for the two-photon absorption rate in this range of intensity is that the transition probability is far from the perturbative regime and close to saturation. We have therefore used Eq. (3) as a power series representing the nonlinear function $P(I)$ calculated in the the- 

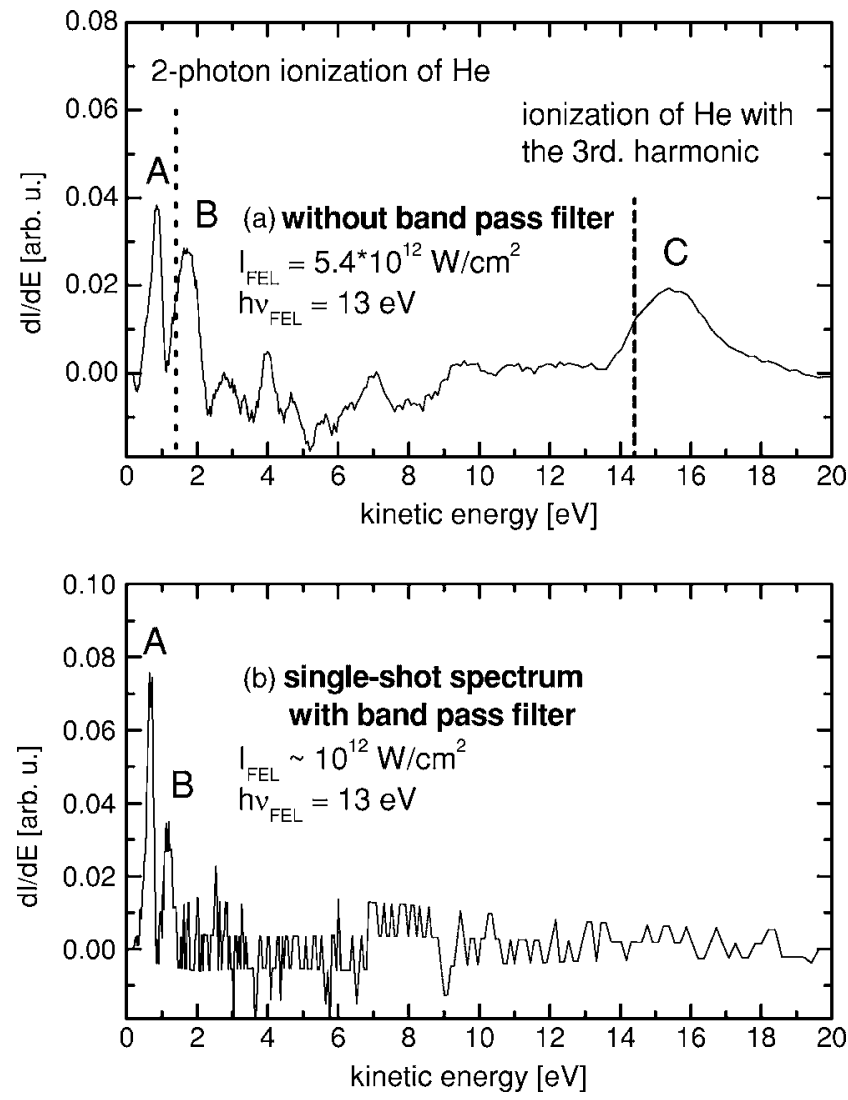

FIG. 5. Electron kinetic energy distribution from He atoms irradiated with VUV FEL light at similar peak intensity of (a) $5.4 \cdot 10^{12} \mathrm{~W} / \mathrm{cm}^{2}$ (without band pass filter), respectively (b) $\sim 10^{12} \mathrm{~W} / \mathrm{cm}^{2}$ (with band pass filter). The calculated energy positions of He photoelectrons are indicated in the figure by dotted lines.

oretical companion paper. This transition probability is shown, in a linear plot, in Fig. 6(b). It is pretty obvious that in the upper limit of the FEL intensities experimentally available, we are very close to a nonmonotonic region of $P(I)$. In the experimentally addressed interval of intensity values, $P(I)$ could be nicely fitted with a third-order polynomial $P(I)=A+B \cdot\left(I+a_{2} I^{2}+a_{3} I^{3}\right)$, with $a_{2}=0.378$ and $a_{3}=-0.047$, see Fig. 6(b). Now, when we integrate the photoelectron generation rate over the interaction region illuminated by the Gaussian FEL beam [Eq. (1)], and scan the FEL intensity by moving the interaction volume a distance $d$ away from the focus (intensity attenuation by $k(d)$, Table II), we find that the detected photoelectron rate is given by:

$$
n_{P E} \cdot k(d)=A^{\prime}+B^{\prime}\left(I_{m} k+\frac{a_{2}}{2}\left(I_{m} k\right)^{2}+\frac{a_{3}}{3}\left(I_{m} k\right)^{3}\right)
$$

$I_{m}$ is the maximum intensity at the center of the beam $(r$ $=0)$ and at the focal plane $(d=0)$, which is kept constant here. The coefficients $A, B, A^{\prime}$, and $B^{\prime}$ are not physically relevant, they just describe different vertical scales and origins for the two physically different quantities $P(I)$ and $n_{P E}$. The function $n_{P E} \cdot k(d)$, with suitably chosen $A^{\prime}$ and $B^{\prime}$, is shown in Fig. 6(a) superimposed on the data points. Given
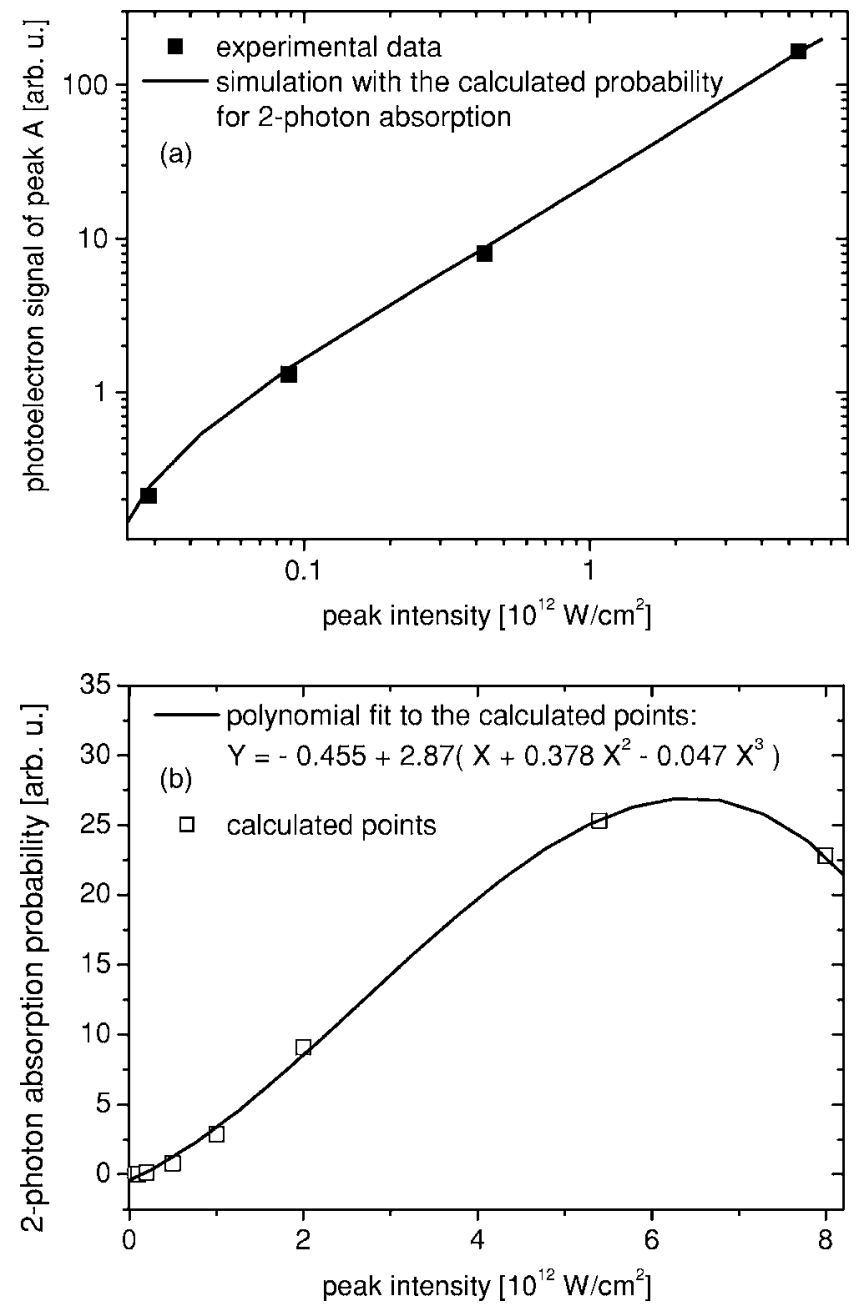

FIG. 6. (a) Dependence of the He photoelectron signal at $1.2 \mathrm{eV}$ kinetic energy (peak A) on the FEL peak intensity in log-log scale. Experimental data (squares) is compared with theoretical prediction for the two-photon absorption (solid line). (b) Probability of twophoton absorption calculated at the indicated FEL intensities after irradiation with a single laser pulse (open squares). The He atom goes from the ground state into a state with one bound electron and one quasi-free electron. A polynomial fit to the theoretically derived values is indicated by the solid line, for details see the text and the companion paper.

the experimental uncertainty, the agreement is very good. We think that the data on Fig. 6 are strong evidence for the presence of two-photon absorption of the fundamental in our helium experiments.

Now we discuss peak $\mathrm{C}$. We attempted to discriminate between the two possible mechanisms by inserting a lowpass indium filter (10\% transmission at the fundamental, negligible transmission at the third harmonic). The resulting photoelectron spectrum is shown in Fig. 5(b). The intensity measured after the filter was approximately $10^{12} \mathrm{~W} / \mathrm{cm}^{2}$. The signal to noise ratio is now poorer, but the peaks A,B are still visible, while peak $\mathrm{C}$ has disappeared. The interpretation of this result is, however, not unique. The first interpretation would say that peak $\mathrm{C}$ was coming from the third harmonic spectral component, now completely suppressed by the indium filter. The second interpretation, however, invokes the 


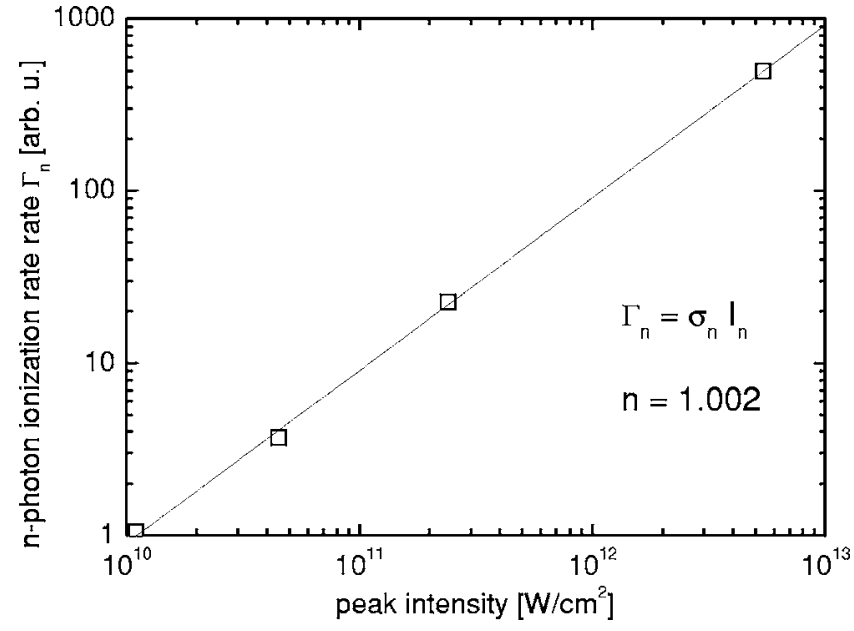

FIG. 7. $n$-photon ionization rate $\Gamma_{n}$ related to peak C in Fig. 5(a) as a function of the FEL peak intensity $I$ in a double-logarithmic scale. The slope of the linear fit $(n=1)$ reflects, that the ionization of $\mathrm{He}$ atoms leading to $15 \mathrm{eV}$-electrons, is governed by third harmonic FEL radiation. The generalized cross section of the ionization process is given by $\xi_{n}$. Data points obtained in the focus and at reduced peak intensities 3,8 , and $15 \mathrm{~mm}$ outside the focus are indicated in the figure.

fact that the three-photon absorption process is roughly proportional to $I^{3}$. Now, the intensity $I$ fell by a factor of 5.4 on insertion of the filter, and the probability for the process is expected to fall by a factor of $5.4^{3} \sim 150$. Since the initial signal to noise ratio for peak $\mathrm{C}$ was already poor [see Fig. $5(\mathrm{a})$, we expect this feature to be lost in the noise on insertion of the indium filter. The line-width of photoelectrons in the single-shot spectrum are slightly smaller than that of the averaged spectrum. This is presumably due to small jitters in the wavelength (different lasing modes of the FEL), timing and focus position on a shot-to-shot basis.

We performed for the intensity dependence of peak $\mathrm{C}$ in the photoemission spectra an analysis analogue to the one applied to peak A. Figure 7 shows the ionization rate $\Gamma_{n}$ of helium atoms leading to peak $\mathrm{C}$, as a function of the VUVFEL peak intensity in a double-logarithmic plot. The slope of the linear fit is in perfect agreement with single photon ionization by third harmonic radiation $(n=1)$. Yet, one has to keep in mind that above-threshold ionization or more specifically the three-photon process will, if at all, take place at maximum pulse intensity in a very small spatial volume. On the other hand, the detector is sensitive to a rather large interaction zone determined by the entrance aperture of $2 \mathrm{~mm}$. Therefore, ionization with third harmonic radiation is very effective on the full $2 \mathrm{~mm}$ beam path (also before and after the focal plane) and consequently this significant detection of "background electrons" dominates peak C.

We now further comment on the photoelectron peaks A, $\mathrm{B}$, and C. First of all, it is important to note that it is not possible to compare the relative intensity of peak A or B with that of peak $C$. This is because the transmission of the spectrometer was calculated without taking into account angular dependence of photoemission, i.e., $s$ - or $p$-symmetric electron wave functions. Additionally, the detector was not

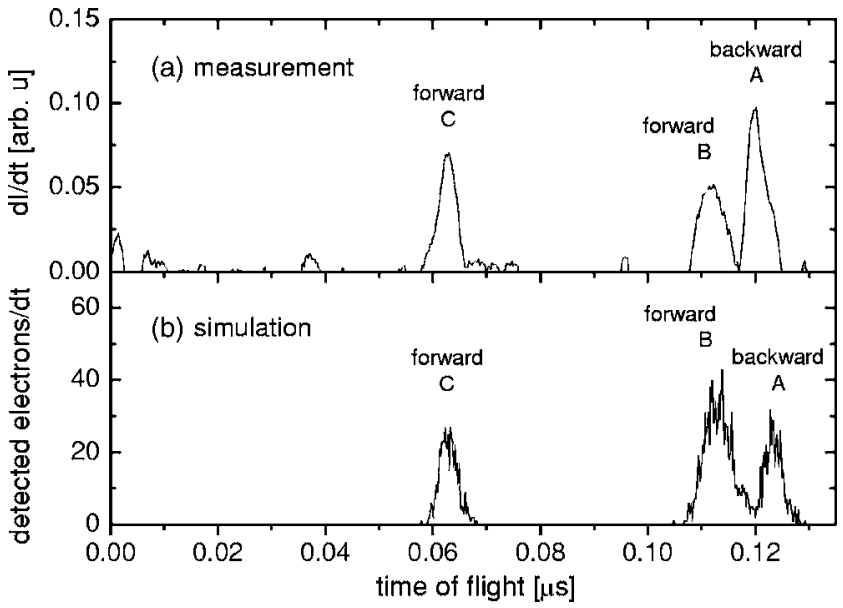

FIG. 8. Comparison between the measured (a) and simulated (b) time-of-flight photoelectron distribution that corresponds to the energy spectrum shown in Fig. 5(a). Electrons ejected into the halfsphere facing towards (forward) and away (backward) from the detector are indicated (for details see the text).

mounted at the "magic" angle. However, the spectra with and without band pass filter [Figs. 5(a) and 5(b)] clearly show, that there is a contribution of two-photon ionization of $\mathrm{He}$ atoms with the fundamental FEL laser frequency at $\sim 10^{12} \mathrm{~W} / \mathrm{cm}^{2}$, which could not be extracted from mass spectroscopy.

The splitting A,B is puzzling. In order to clarify its origin, we have simulated the time-of-flight distribution of photoelectrons corresponding to the energy spectrum shown in Fig. 5(a) by using an electron optics ray-tracing program. In the calculation of the electron trajectories, the measured center kinetic energy and width of only two peaks, $B$ and $C$, are taken as input parameters. For computational reasons, an isotropic emission of $\sim 640.000$ electrons is assumed for each distribution. In Figs. 8(a) and 8(b), the results of the simulations [Fig. 8(b)] are compared with the measured flight time distribution of photoelectrons [Fig. 8(a)] after subtracting the background spectrum recorded without gas flow. From this analysis, we can clearly identify the origin of the third photoelectron peak, A. It corresponds to electrons with a mean kinetic energy of $1.7 \mathrm{eV}$, which are ejected in the half-sphere facing away from the detector ("backward electrons"). Due to the electric field of $10 \mathrm{~V}$, which is applied between the grounded aperture and the acceleration plate, those electrons are "pushed" towards the detector on a somewhat longer time scale $(\Delta t \sim 10 \mathrm{~ns})$. The observed splitting in case of A and $\mathrm{B}$ (instead of an intuitively expected broadening) is due to the fact, that electrons ejected perpendicularly to the TOF axis are not collected. They hit the aperture of the field-free drift tube. In the light of the previous discussion on the origin of the A-B splitting, it is surprising that peak $C$ is not split as well. This can be understood in the following way. The acceleration plate has a $3 \mathrm{~mm}$ hole in its center. Consequently, "backward" electrons with kinetic energies $\geqslant 5 \mathrm{eV}$ that are emitted within a small cone, almost on axis, are lost for the detection. They fly through the acceleration plate. From a detailed analysis of the electron trajectories, we derived that for the distribution of photoelectrons with a mean 


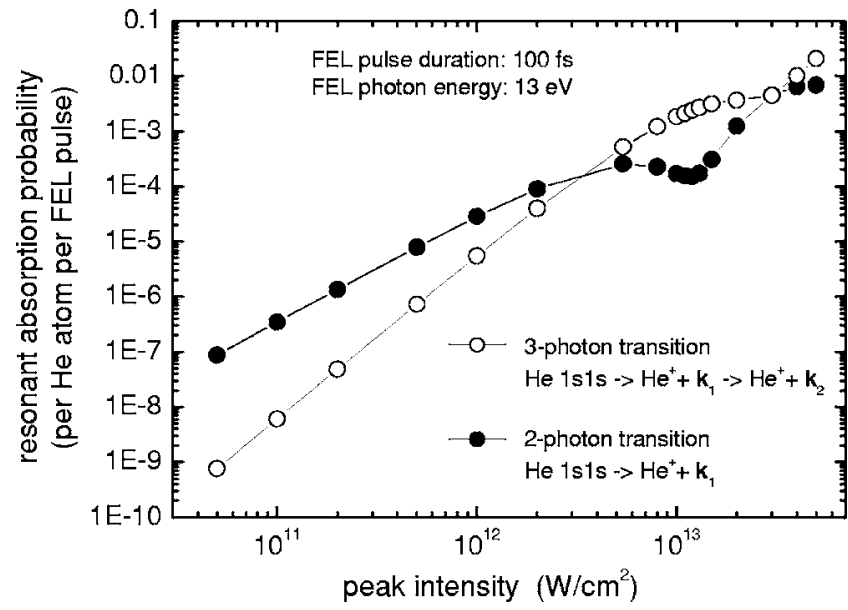

FIG. 9. Peak intensity dependence of the resonant absorption probability for two- and three-photon transition from the He $1 s 1 s$ singlet ground state into quasifree $\mathrm{He}^{+}+\mathbf{k}_{\mathbf{1}}$, respectively, $\mathrm{He}^{+}+\mathbf{k}_{\mathbf{2}}$ states. VUV-FEL parameters are given in the figure.

kinetic energy of $15.4 \mathrm{eV}$ (peak C) only a few "forward electrons" reach the MCP, namely those that are emitted towards the detector within a small opening cone angle. All other electrons hit either the aperture, the acceleration plate, or fly through the hole.

Finally, we put these experimental results in perspective with the calculation in the companion paper. It consists in the numerical solution of the time-dependent Schrödinger equation using the fully quantized field and a limited but representative set of basis states [25]. As a key result, Fig. 9 shows the peak intensity dependence of the resonant absorption probability for two- and three-photon transitions with the fundamental of the VUV-FEL. As input laser parameters for the calculation, we took those of the measurement presented in Fig. 5(a). The peak intensity was varied in the range of $5 \cdot 10^{10}-5 \cdot 10^{13} \mathrm{~W} / \mathrm{cm}^{2}$. The two-photon transition from the He $1 s 1 s$ singlet ground state into the $\mathrm{He}^{+}+\mathbf{k}_{\mathbf{1}}$ state, with one electron bound to a $Z=2$ nucleus plus a quasifree electron with a kinetic energy $\hbar^{2} k_{1}^{2} / 2 m=1.4 \mathrm{eV}$, grows initially with a slope close to two and shows a rather complicated behavior above $\sim 5 \cdot 10^{12} \mathrm{~W} / \mathrm{cm}^{2}$ due to interference effects. The resonant absorption probability for the threephoton transition into the $\mathrm{He}^{+}+\mathbf{k}_{\mathbf{2}}$ state $\left(\hbar^{2} k_{2}^{2} / 2 m\right.$ $=14.4 \mathrm{eV}$ ) starts of very small but competes with the $\mathrm{He}^{+}$ $+\mathbf{k}_{\mathbf{1}}$ population at $\sim 3 \cdot 10^{12} \mathrm{~W} / \mathrm{cm}^{2}$. From the calculation, it is expected that at the highest peak intensity experimentally under investigation $\left(5.4 \cdot 10^{12} \mathrm{~W} / \mathrm{cm}^{2}\right)$, the three-photon process should contribute to the ionization of He atoms, both in the ion yield (Fig. 3) and the electron yield [Fig. 5(a)]. The calculated probabilities for these two processes, for $100 \mathrm{fs}$ FEL pulses and a peak intensity of $5.4 \cdot 10^{12} \mathrm{~W} / \mathrm{cm}^{2}$ in the fundamental and $5.4 \cdot 10^{9} \mathrm{~W} / \mathrm{cm}^{2}$ in the third harmonic, are comparable within $20 \%$.

The results here reported give evidence of nonlinear phenomena in He gas at thresholds which, for $13 \mathrm{eV}$ VUV light, are much smaller than for IR or visible light. The photoemission spectra show unambiguously the presence of twophoton absorption at thresholds of $10^{12} \mathrm{~W} / \mathrm{cm}^{2}$. The analysis of a photo-emission feature attributable to three-photon ab- sorption of the fundamental was rendered inconclusive due to the presence of a third harmonic spectral component in the FEL beam. These measurements point towards very interesting future experiments with fourth-generation FEL light sources, namely pump-probe spectroscopy using different higher harmonics of the FEL at a wavelength capable of providing atomic resolution. One of the major advantages for the investigation of ultra-fast processes in matter on a fs timescale is that the radiation pulses are naturally synchronized. With the help of optical components, such as filters, gratings, and delay-lines, etc., it will be feasible, for instance, to prepare a specific state of matter with a first harmonic pump pulse and to probe its time evolution with a third harmonic probe pulse. This will open the door to fs timeresolved spectroscopy with atomic resolution.

\section{CONCLUSION}

We have presented time-of-flight mass and photoelectron spectroscopy of He atoms in order to investigate the interaction with intense vacuum-ultraviolet (VUV) light from a free-electron laser (FEL). Irradiation of the atomic beam with fs-pulses at a peak intensity of $10^{10}-10^{13} \mathrm{~W} / \mathrm{cm}^{2}$ results in efficient ionization of He atoms, as seen by a strong signal in the time-of-flight mass spectra. The resulting $\mathrm{He}^{+}$ ions seem to be mainly created by single photon absorption of third harmonic FEL radiation. In addition, comprehensive studies give evidence that nonlinear two-photon absorption is the source of two sharp features in the photo-electron spectrum of He atoms. This is in quantitative agreement with the numerical solution of the time-dependent Schrödinger equation, as described in the companion paper. At high peak intensity $\left(\geqslant 10^{12} \mathrm{~W} / \mathrm{cm}^{2}\right.$ the calculation predicts a large probability for two and three-photon transitions from the $\mathrm{He}$ ground state into a continuum state. These correspond to above threshold ionization peaks in the photoelectron spectra. The one due to two-photon absorption was quantitatively fitted to the calculated transition probability. The one attributable to three-photon absorption, unfortunately, could not be separated experimentally from the dominant signal of "background electrons" due to the absorption of third harmonic FEL light. This is presumably due to the relatively long laser beam path-with efficient single photon ionization-covered by the spectrometer. The experimental results indicate that the photon flux in higher modes of the VUV-FEL is sufficient to perform single-shot spectroscopy on gas phase samples. This might open the door to very interesting two-color pump-probe experiments with these powerful light sources at even shorter wavelength, namely fs time-resolved spectroscopy with atomic resolution, using different naturally synchronized higher harmonics of an FEL.

\section{ACKNOWLEDGMENTS}

We thank A. Swiderski for technical support in the design and construction of the experiment and the TTF team at DESY for running the accelerator, working on the FEL and the photon diagnostics. This work was supported by the DFG. 
[1] P. Agostini, F. Fabre, G. Mainfray, G. Petite, and N. K. Rahman, Phys. Rev. Lett. 42, 1127 (1979).

[2] M. Gavrila, Atoms in Intense Laser Fields (Academic, New York, 1992).

[3] J. Wassaf, V. Veniard, R. Taeb, and A. Maquet, Phys. Rev. A 67, 053405 (2003).

[4] G. G. Paulus, W. Nicklich, H. Xu, P. Lambropoulos, and H. Walther, Phys. Rev. Lett. 72, 2851 (1994).

[5] B. Walker, B. Sheehy, K. C. Kulander, and L. F. DiMauro, Phys. Rev. Lett. 77, 5031 (1996).

[6] G. G. Paulus and H. Walther, in Ref. [26], pp. 83-97 (2001).

[7] M. P. Hertlein, P. H. Bucksbaum, and H. G. Muller, J. Phys. B 30, L197 (1997).

[8] P. Hansch, M. A. Walker, and L. D. V. VanWoerkom, Phys. Rev. A 55, R2535 (1997).

[9] G. G. Paulus, F. Grasbon, H. Walther, R. Kopold, and W. Becker, Phys. Rev. A 64, 021401(R) (2001).

[10] J. Wassaf, V. Veniard, R. Taeb, and A. Maquet, Phys. Rev. Lett. 90, 013003 (2003).

[11] U. Johann, T. S. Luk, H. Egger, and C. K. Rhodes, Phys. Rev. A 34, 1084 (1986).

[12] T. S. Luk, T. Graber, H. Jara, U. Johann, K. Boyer, and C. K. Rhodes, J. Opt. Soc. Am. B 4, 847 (1987).

[13] V. Ayvazyan et al., Phys. Rev. Lett. 88, 104802 (2002).

[14] H. Wabnitz et al., Nature (London) 420, 482 (2002).
[15] J. Schulz, H. Wabnitz, T. Laarmann, A. de Castro, P. Gürtler, W. Laasch, A. Swiderski, and T. Möller, Nucl. Instrum. Methods Phys. Res. A 507, 572 (2003).

[16] T. Laarmann, H. Wabnitz, T. Laarmann, A. R. B. de Castro, P. Gürtler, W. Laasch, and T. Möller, Phys. Rev. Lett. 92, 143401 (2004).

[17] T. Laarmann, M. Rusek, H. Wabnitz, J. Schulz, A. de Castro, P. Gürtler, W. Laasch, and T. Möller, Phys. Rev. Lett. 95, 063402 (2005).

[18] V. Ayvazyan et al., Eur. Phys. J. D 20, 149 (2002).

[19] B. Faatz et al., DESY Print TESLA-FEL 2001-09, pp. 62-67 (2001).

[20] E. L. Saldin, E. A. Schneidmiller, and M. V. Yurkov, The Physics of Free-Electron Lasers (Springer-Verlag, Berlin, Heidelberg, New York, 2000).

[21] E. Saldin (private communications).

[22] S. Laulan and H. Bachau, Phys. Rev. A 68, 013409 (2003).

[23] L. V. Keldysh, Sov. Phys. JETP 20, 1307 (1965).

[24] M. Protopapas, C. H. Keitel, and P. H. Knight, Rep. Prog. Phys. 60, 389 (1997).

[25] A. de Castro, T. Laarmann, J. Schulz, H. Wabnitz, and T. Möller, following paper, Phys. Rev. A 72, 023410 (2005).

[26] D. Batani et al., Atoms, Solids and Plasmas in Super-Intense Laser Fields (Kluwer Academic/Plenum Publishers, New York, 2001). 